УДК 538.9:536.6

\title{
ТЕПЛОПРОВОДНОСТЬ ПОЛИМЕРНЫХ МИКРО- И НАНОКОМПОЗИТОВ НА ОСНОВЕ ПОЛИЭТИЛЕНА ПРИ РАЗЛИЧНЫХ МЕТОДАХ ИХ ПОЛУЧЕНИЯ
}

\author{
Фиалко Н.М. ${ }^{1}$, член-корреспондент НАН Украины, Динжос Р.В. ${ }^{2}$, канд. физ.-мат. наук, \\ Шеренковский Ю.В.', канд. техн. наук, Меранова Н.О. ${ }^{1}$, канд. техн. наук, \\ Навродская P.A. ${ }^{1}$, канд. техн. наук \\ ${ }^{1}$ Институт технической теплофизики НАН Украины, ул. Желябова, 2 а, Киев, 03680, Украина \\ ${ }^{2}$ Николаевский наииональный университет им. В.А.Сухомлинского, ул. Никольскал, 24, Николаев, 54030, Украина
}

Наведено результати порівняльного аналізу теплопровідних властивостей полімерних мікро- і нанокомпозитів на основі поліетилену, одержаних із застосуванням методів, що базуються на змішуванні компонентів у сухому вигляді або розплаві полімерної матриці. Для поліетилену, наповненого вуглецевими нанотрубками i частками алюмінію, представлено дані щодо впливу методів синтезу композитів на величини перколяційних порогів, концентраційну залежність коефіцієнтів теплопровідності тощо.
Приведены результаты сравнительного анализа теплопроводящих свойств полимерных микро- и нанокомпозитов на основе полиэтилена, полученных с применением методов, базирующихся на смешении компонентов в сухом виде или расплаве полимерной матрицы. Для полиэтилена, наполненного углеродными нанотрубками и частицами алюминия представлены данные влияния методов синтеза композитов на величины перколяционных порогов, концентрационную зависимость коэффициентов теплопроводности и т.д.
The results of a comparative analysis of the heat conductivity properties of polymer micro- and nanocomposites based on polyethylene obtained using methods based on the mixing of components in a dry form or a melt of a polymer matrix are submitted. For polyethylene, filled with carbon nanotubes and aluminum particles, data on the effect of methods of synthesis of composites on the values of percolation thresholds, the concentration dependence of the coefficients of heat conductivity, etc. are presented.

Библ. 15, рис. 4.

Ключевые слова: полимерные микро- и нанокомпозиты, коэффициент теплопроводности, методы синтеза композитов, углеродные нанотрубки.

$\Delta \lambda$ - отличие коэффициентов теплопроводности композитов, которые получены с использованием методов, основанных на смешении компонентов в расплаве полимера и в сухом виде;

$\lambda$ - коэффициент теплопроводности;

\section{Введение}

Полимерные микро- и нанокомпозиты находят все более широкое применение в конструкциях различного оборудования. Это в значительной мере обусловлено тем, что данный класс материалов обладает целым рядом уникальных технологических и физико-механических характеристик [1-8]. Среди таких характеристик особо выделяется широкий спектр теплопроводящих свойств полимерных композитов, от их низкотеплопроводных до высокотеплопроводных модификаций. В настоящей статье большое внимание уделяется исследованию коэффициента теплопроводности полимерных композиционных материалов при различных типах наполнителей, разных величинах их массовых долей и пр.

На теплопроводящие свойства полимерных микрои нанокомпозитов, как известно, существенное влияние могут оказывать методы их синтеза [9-12]. В этой связи важной является задача установления зависимости данных свойств от метода получения композиционного материала. $\omega$ - массовая доля наполнителя;

$\omega_{1}$ и $\omega_{2}-$ первый и второй порог перколяции; УНТ - углеродные нанотрубки.

\section{Постановка задачи и методика проведения исследований}

Настоящая статья посвящена сравнительному анализу теплопроводящих свойств полимерных микро- и нанокомпозитов, полученных с использованием различных методов.

Рассмотрению подлежали следующие методы синтеза полимерных композитов: во-первых, метод, в котором смешение компонентов, находящихся в сухом виде, производится с использованием магнитной мешалки и ультразвукового диспергатора при дальнейшем горячем прессовании полученной композиции (метод $A$ ); и вовторых, метод, основанный на смешении компонентов в расплаве полимера с применением дискового экструдера при дальнейшем придании композиту необходимой формы способом горячего прессования (метод $B$ ).

Эти методы использовались для синтеза композитов на основе частично-кристаллического полиэтилена с применением в качестве наполнителей УНТ или микрочастиц алюминия. Применяемые в ходе исследований 
углеродные нанотрубки изготавливались методом CVD (англ. Chemical vapor deposition - химическое парофазное осаждение). Содержание минеральных примесей в них составляло $\sim 0,1 \%$. Удельная площадь поверхности УНТ, определенная адсорбцией $N_{2}$, равнялась $190 \mathrm{~m}^{2} / \Gamma$. Внешний диаметр УНТ, определенный с помощью метода малоуглового рассеяния рентгеновских лучей, составлял 20 нм, длина - (1..5) мкм, толщина стенок $\sim 5$ нм $[13,14]$. Производитель углеродных трубок ООО «Спецмаш».

Используемые в качестве наполнителя микрочастицы алюминия были получены из алюминиевых опилок посредством их растирания в шаровой мельнице до образования частиц размером $(0,5 \ldots 1)$ мкм. Коэффициент теплопроводности полимерных композитов определялся с использованием прибора ИТ- $\lambda-400$ [15].
При оценке себестоимости разрабатываемых композитов стоимость их компонентов принимались по данным Лондонской товарной биржи.

\section{Изложение основного материала исследований}

Характерные результаты выполненных экспериментальных исследований представлены на рис. 1, 2. Рис. 1 иллюстрирует концентрационные зависимости коэффициента теплопроводности для полимерных микро- и нанокомпозитов, полученных с применением методов $A$ и $B$, которые базируются на смешении компонентов в сухом виде и в расплаве полимера соответственно. Как видно, для данных методов существенно отличаются значения коэффициентов теплопроводности композиционных материалов, величины перколяционных порогов, характер зависимости $\lambda=f(\omega)$ и пр.


Рис. 1. Зависимость от массовой доли наполнителя коэффициентов теплопроводности полимерных композитов на основе полиэтилена, полученных с использованием методов смешения компонентов в сухом виде $(1,2)$ и в расплаве полимера $(3,4)$ при наполнении полимерной матрицы микрочастицами алюминия $(1,3)$ и УНТ $(2,4):$ a), б) -линейная и логарифмическая икала по оси ординат соответственно.

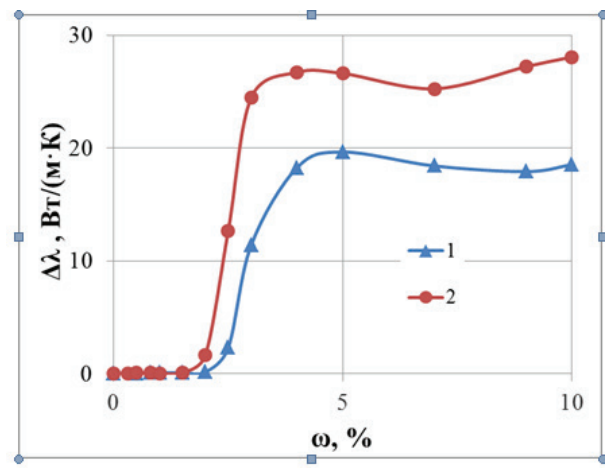

Рис. 2. Отличие коэффициентов теплопроводности композиционныхматериалов $А$ на основе полиэтилена, отвечающих различным методам их получения при наполнении полимерной матрицы микрочастицами алюминия (1) и углеродными нанотрубками (2). 
Что касается отличий $\Delta \lambda$ в значениях коэффициентов теплопроводности рассматриваемых композитов при использовании разных методов их получения, то согласно приведенным данным они могут быть весьма значительными. При этом во всем исследуемом диапазоне изменения $\omega$ большие величины $\lambda$ отвечают композиционным материалам, полученным на основе метода $B$. Представленные результаты свидетельствуют также о том, что указанные отличия в значениях $\lambda$ существенно зависят от типа наполнителя и его массовой доли $\omega$. Как видно из рис. 2 , отличия $\Delta \lambda$ для полиэтилена, наполненного УНТ, оказываются более существенными, чем при его наполнении $A l$. Так, в первом случае максимальные значения $\Delta \lambda$ составляют $28,1 \mathrm{BT} /(\mathrm{M} \cdot \mathrm{K})$, а во втором лишь 19,7 Вт/(м·К).

Относительно зависимостей величины $\Delta \lambda$ от массовой доли наполнителя, то, как видно из рис. 2, они качественно одинаковы для полиэтилена, наполненного алюминием и УНТ. А именно, при низких значениях $\omega$ отличия $\Delta \lambda$ пренебрежимо малы. Далее с увеличением $\omega$ они существенно повышаются, достигая локального максимума, и затем несколько снижаются при дальнейшем незначительном росте. То есть расхождение величин $\lambda$ композитов, отвечающих разным методам их получения, оказываются весьма существенными практически во всем диапазоне изменения массовой доли наполнителя, исключая ее низкие значения.

Важно также подчеркнуть, что методы получения композитов оказывают существенное влияние на максимальные значения их коэффициента теплопроводности, которые могут быть достигнуты в рамках рассматриваемого диапазона изменения массовой доли наполнителя. Как следует из приведенных данных, при использовании метода $B$ максимальное значение $\lambda$ для полиэтилена, наполненного алюминием, превышает соответствующее значение, отвечающее методу $A$, в 3,2 раза. Для полиэтилена, наполненного УНТ, это превышение составляет 2,6 раза.

Представленные экспериментальные данные свидетельствуют о том, что особенности проявления эффектов резкого изменения $\lambda$ композитов при определенных значениях $\omega$ существенно зависят от метода их получения. Как видно из рис. 16 , зависимости $\lambda=f(\omega)$ характеризуются наличием двух скачков коэффициентов теплопроводности, которые отвечают величинам $\omega$, называемым перколяционными порогами. Достижению первого из указанных порогов соответствует образование перколяционных кластеров из частиц наполнителя, достижению второго - формирование перколяционной сетки. Данные перколяционные структуры представляют собой высокотеплопроводные среды, в большой мере ответственные за теплопроводящие свойства композитов в целом.

Как видно из рис. 1б, для полиэтилена, наполненного как $A l$, так и УНТ, значения перколяционных порогов оказываются меньшими в случае применения метода $B$. При использовании в качестве наполнителя микрочастичек $A l$ первый перколяционный порог $\omega_{1}$ равняется $0,59 \%$ и $0,55 \%$, а второй $\omega_{2}$ порог $2,96 \%$ и $1,98 \%$ для методов $A$ и $B$ соответственно. В случае же применения в качестве наполнителя УНТ первый перколяционный порог отвечает $\omega_{1}=0,48 \%$, а второй $\omega_{2}=2,09 \%$ при сухом методе смешения компонентов (метод $A$ ) и $\omega_{1}=0,33 \%$ и $\omega_{2}=1,55 \%$ при смешении компонентов в расплаве полимера (метод $B)$.

Из данных, приведенных на рис. $1 a$, следует, что методы получения композитов оказывают заметное влияние на характер зависимости $\lambda=f(\omega)$. Во-первых, для композитов, полученных на основе метода $A$, заметный рост коэффициента теплопроводности $\lambda$ начинается при больших, чем для метода $B$ значениях $\omega$, отвечающих второму перколяционному порогу. И далее, с повышением массовой доли наполнителя коэффициенты теплопроводности монотонно возрастают.

При получении же композитов с использованием метода $B$ имеет место весьма резкое увеличение $\lambda$ при $\omega>\omega_{2}$, которое при некотором значении $\omega=\omega$, сменяется существенно менее интенсивным ростом. В случае наполнения полиэтилена микрочастицами алюминия $\omega_{\mathrm{c}} \approx 4 \%$, а при наполнении его УНТ $\omega_{\mathrm{c}} \approx 3 \%$.

Что касается описанных закономерностей влияния методов получения рассматриваемых полимерных композитов на их теплопроводящие свойства, то они непосредственно связаны со степенью равномерности распределения наполнителя в полимерной матрице. Более высокие значения коэффициентов теплопроводности композитов, полученных с использованием метода $B$, обусловлены большей равномерностью распределения наполнителя в расплаве полимера. Указанная повышенная равномерность служит причиной более эффективного формирования перколяционных структур, являющихся своеобразными высокотеплопроводящими каналами. Данное обстоятельство и определяет увеличение коэффициентов теплопроводности композитов, полученных по методу $B$.

Как уже отмечалось, влияние метода получения композитов на их теплопроводящие свойства оказывается более существенным для полиэтилена, наполненного УНТ, чем при его наполнении микрочастицами алюминия. Это объясняется тем, что формирование перколяционных структур из УНТ ввиду их значительной относительной длины в большей мере зависит от равномерности распределения наполнителя в полимерной матрице. Так что повышение степени равномерности распределения в полимерной матрице УНТ в случае их получения по методу $B$ приводит к более эффективному формированию перколяционных кластеров и сеток.

Проведенный сравнительный анализ методов получения полимерных микро- и нанокомпозитов касается сопоставления только их теплопроводящих свойств. Очевидно, в общем случае при выборе того или иного метода могут приниматься во внимание различные критерии эффективности их использования, такие как объемы применения композитов, престижная эффективность («результат любой ценой»), себестоимость их получения и т.д. Что касается себестоимости собственно реализации метода, то она заметно выше для 
метода $B$ ввиду наличия процесса проплавления полимера. Однако стоимость компонентов композиционного материала при заданном значении $\lambda$ оказывается большей в случае метода $A$ в связи с большей массовой долей дорогостоящего наполнителя ( $A l$ или УНТ). Таким образом, в общей себестоимости получения полимерных композитов фигурируют два конкурирующих фактора. Первый из них, касающийся себестоимости собственно технологии, и второй - себестоимости компонентов композиционного материала. При доминировании того или иного фактора более предпочтительным оказывается соответствующий метод.

На рис. 3, 4 представлены зависимости себестоимости рассматриваемых композитов от величины коэффициента теплопроводности в линейной и логарифмической координатах. Как видно, в плане меньшей себестоимости композитов метод $A$ является более эффективным лишь при низких значениях $\lambda$, отвечающих небольшим массовым долям наполнителя.
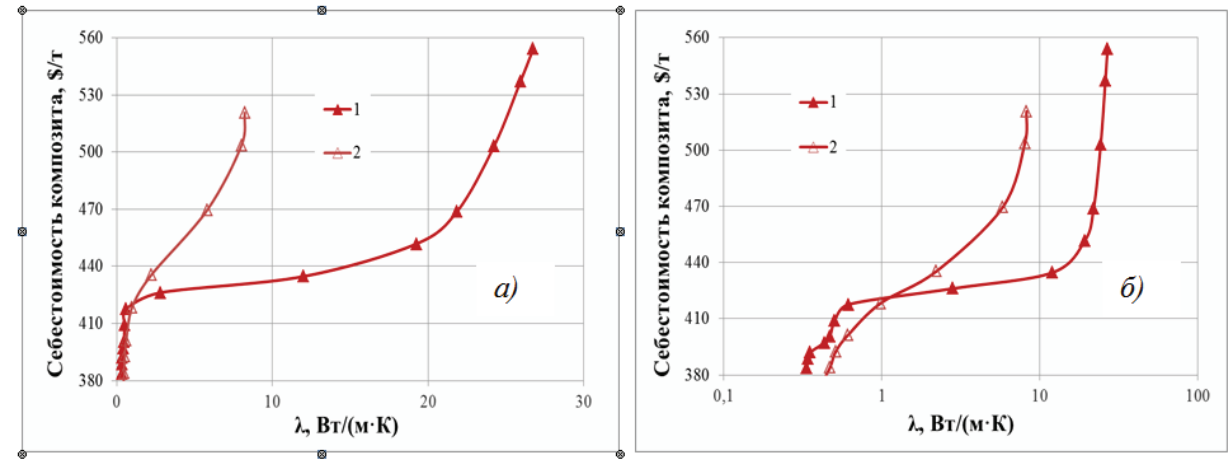

Рис. 3. Себестоимость композиционных материалов на основе полиэтилена, наполненного микрочастицами алюминия, для различных методов получения композитов: метод В (1); метод А (2); а), б) - линейная и логарифмическая икала по оси абсцисс соответственно.
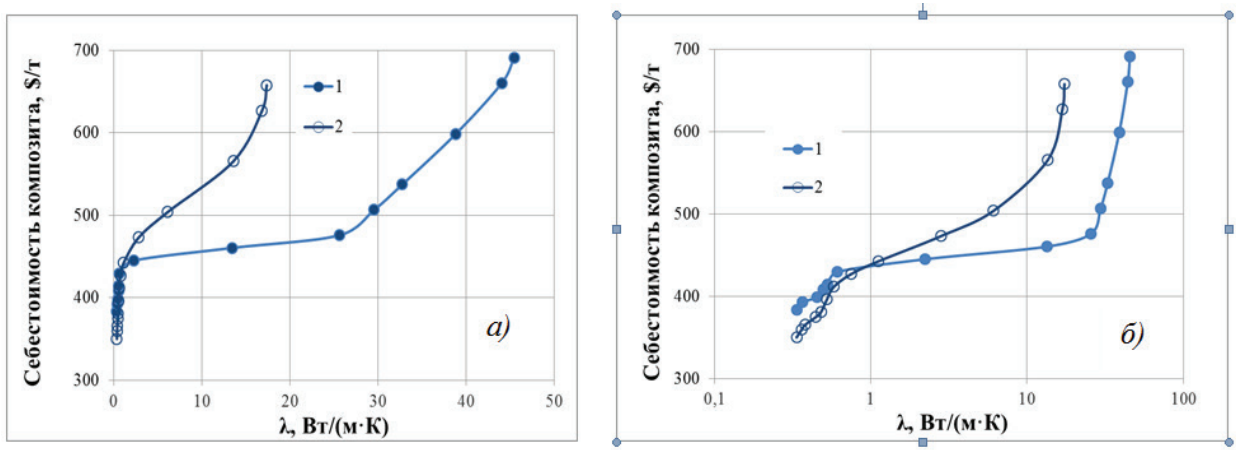

Рис. 4. Себестоимость композиционных материалов на основе полиэтилена, наполненных углеродныли нанотрубками, для различных методов получения композитов:

метод В (1); метод А; а), б) -линейная и логарифмическая икала по оси абсцисс соответственно.

\section{Выводы}

1. На основе экспериментальных исследований проведен сравнительный анализ характеристик полимерных микро- и нанокомпозиционных материалов на основе полиэтилена в условиях использования двух методов их получения, которые базируются на смешении компонентов в сухом виде (метод $A$ ) и в расплаве полимерной матрицы (метод $B)$.

2. Построены концентрационные зависимости ко- эффициентов теплопроводности для полиэтилена, наполненного УНТ или микрочастицами алюминия. При этом показано, что:

- $\quad$ применение метода $B$ может обеспечить получение микро- и нанокомпозитов с существенно более высокими теплопроводящими свойствами;

- при наполнении полиэтилена УНТ эффект повышения его коэффициента теплопроводности, обусловленный применением метода $B$, оказывается более 
значительным, чем в случае использования в качестве наполнителя микрочастиц $A l$;

- для метода $A$ оба скачка коэффициента теплопроводности рассматриваемых композитов на кривой $\lambda=f(\omega)$ отвечают большим значениям массовой доли наполнителя $\omega_{1}$ и $\omega_{2}$.

3. Выполнена оценка себестоимости полимерных композитов, полученных с использованием методов, основанных на смешении компонентов в сухом виде и в расплаве полимерной матрицы. Установлено, что данная себестоимость определяется в большой мере двумя конкурирующими факторами, каждый из которых отражает преимущества одного из методов, а именно, меньшую при фиксированном значении $\lambda$ стоимость компонентов композита для метода $B$, и более низкую стоимость собственно реализации технологии для метода $A$. Показано, что по себестоимости рассматриваемых композитов метод $B$ является предпочтительным во всем диапазоне изменения массовой доли наполнителя, исключая ее весьма низкие значения, где целесообразно использование метода $A$.

\section{ЛИТЕРАТУРА}

1. Han Z., Fina A. Thermal conductivity of carbon nanotubes and their polymer nanocomposites: a review// Prog. Polym. Sci. - 2011. - Vol. 36. - P. 914-944.

2. Improving thermal conductivity while retaining high electrical resistivity of epoxy composites by incorporating silicacoated multiwalled carbon nanotubes / Wei Cui, Feipeng Du, Jinchao Zhao, Wei Zhang, Yingkui Yang, Xiaolin Xie, Yiu-Wing Mai // Carbon. - 2011. - Vol. 49. - P. 495-500.

3. A large increase in the thermal conductivity of carbon nanotube/polymer composites produced by percolation phenomena / Su Yong Kwon, Il Min Kwon, Yong-Gyoo Kim, Sanghyun Lee, Young-Soo Seo// Carbon.2013. - Vol. 55. - P. 285-290.

4. Polyethylene nanofibres with very high thermal conductivities / Sheng Shen, Asegun Henry, Jonathan Tong, Ruiting Zheng and Gang Chen // Nature nanotechnology. 2010. - Vol. 5. - P. 251-255.

5. Carbon nanotube-polymer composites: Chemistry, processing, mechanical and electrical properties / Zdenko Spitalsky, Dimitrios Tasis, Konstantinos Papagelis, Costas Galiotis // Progress in Polymer Science. - 2010. - Vol. 35. P. 357-401.
6. Hernandez-Montelongo J. Porous siliconcyclodextrin based polymer composites for drug delivery applications / J. Hernandez-Montelongo, N. Naveas, S. Degoutin, N. Tabary, F. Chai, V. Spampinato, G. Ceccone, F. Rossi, V. Torres-Costa, M. Manso-Silvan, B. Martel. Carbohydrate Polymers. - 2014. - Vol. 110. - P. 238-252.

7. Diaz-Bleis D. Thermal characterization of magnetically aligned carbonyl iron/agar composites / D. Diaz-Bleis, C. Vales-Pinzón, Y. Freile-Pelegrín, J.J. Alvarado-Gil. - Carbohydrate Polymers. - 2014. - Vol. 99. P. 84-90.

8. Samanvaya S. Polymer-Particle Composites: Phase Stability and Applications in Electrochemical Energy Storage / Samanvaya Srivastava, Jennifer L. Schaefer, Zichao Yang, Zhengyuan Tu and Lynden A. Archer. Advansed Material. - 2014. - Vol. 26. - P. 201-234.

9. Sathyanarayana $S$. Thermoplastic Nanocomposites with Carbon Nanotubes / S. Sathyanarayana, C. Hübner. Structural Nanocomposites. - 2013. - P. 19-60.

10. Huang Y. Y. Dispersion of Carbon Nanotubes: Mixing, Sonication, Stabilization, and Composite Properties / Yan Yan Huang, Eugene M. Terentjev. - Polymers. - 2012. V. 4.- P. 275-295.

11. Peng-Cheng Ma. Dispersion and functionalization of carbon nanotubes for polymerbased nanocomposites: A review / Peng-Cheng Ma, Naveed A. Siddiqui, Gad Marom, Jang-Kyo Kim. - Composites: Part A. - 2010. - V. 41.P. $1345-1367$.

12. Дінжос Р. В. Вплив методу введення наповнювача на теплофізичні властивості систем на основі термопластичних полімерів та вуглецевих нанотрубок / Р. В. Дінжос, Е. А. Лисенков, Н. М. Фіалко, В. В. Клепко // Фізична інженерія поверхні. - 2014. - Т.12, №4. C. 446-453.

13. Малежик А.В., Семенцов Ю.И., Янченко В.В. Синтез углеродных нанотрубок методом каталитического разложения // Журнал прикладной химии. - 2005. T.78. - C. 938-943.

14. Структура многослойных углеродных нанотрубок, полученных каталитическим разложением этилена на наночастицах никеля / Н.В. Лемеш, Э.А. Лысенков, Ю.П. Гомза и др. // Украинский химический журнал. 2010. - T. 76, №5. - C. 29-36.

15. Giovanni A. L. A Steady-State Apparatus to Measure the Thermal Conductivity of Solids // Int. J. Thermophys. 2008. - Vol. 29. - P. 664-677. 


\section{HEAT CONDUCTIVITY OF POLYMERIC MICRO- AND NANOCOMPOSITES BASED ON POLYETHYLENE AT VARIOUS METHODS OF THEIR PREPARATION}

Fialko N.M. ${ }^{1}$, Dinzhos R.V. ${ }^{2}$, Sherenkovskiy Yu.V. ${ }^{1}$, Meranova N.O. ${ }^{1}$, Navrodskaya R.A. ${ }^{1}$

${ }^{1}$ Institute of Engineering Thermophysics, National Academy of Sciences of Ukraine,

2a, Zhelyabova str., Kyiv, 03680, Ukraine

${ }^{2}$ Nikolaev National University named after V.A.

Sukhomlinskiy, 24, Nikolska str., Mykolaev, 540030, Ukraine

Key words: polymeric micro- and nanocomposites, heat conductivity, methods of synthesis of composites, carbon nanotubes.

The results of a comparative analysis of the heat conductivity properties of polymer micro- and nanocomposites based on polyethylene obtained using methods based on the mixing of components in a dry form or a melt of a polymer matrix are submitted. For polyethylene, filled with carbon nanotubes and aluminum particles, data on the effect of methods of synthesis of composites on the values of percolation thresholds, the concentration dependence of the coefficients of heat conductivity, etc. are presented. Based on the theory of percolation, the dependence of the heat conductivity coefficients of the polymer composites on the methods of their synthesis has been interpreted. The questions of an estimation of the cost price of investigated polymeric composites at use of different methods of their reception are considered.

Reference 15 , figures 4 .

1. Han Z., Fina A. Thermal conductivity of carbon nanotubes and their polymer nanocomposites: a review, Prog. Polym. Sci., 2011, Vol. 36, p. 914-944. (Eng.)

2. Wei Cui, Feipeng Du, Jinchao Zhao, Wei Zhang, Yingkui Yang, Xiaolin Xie, Yiu-Wing Mai. Improving thermal conductivity while retaining high electrical resistivity of epoxy composites by incorporating silicacoated multiwalled carbon nanotubes, Carbon, 2011, Vol. 49,- p. 495500. (Eng.)

3. Su Yong Kwon, Il Min Kwon, Yong-Gyoo Kim, Sanghyun Lee, Young-Soo Seo. A large increase in the thermal conductivity of carbon nanotube/polymer composites produced by percolation phenomena, Carbon, 2013, Vol. 55, p. 285-290. (Eng.)

4. Sheng Shen, Asegun Henry, Jonathan Tong, Ruiting Zheng, Gang Chen. Polyethylene nanofibres with very high thermal conductivities, Nature nanotechnology, 2010, Vol. 5, p. 251-255. (Eng.)

5. Spitalsky Zdenko, Tasis Dimitrios, Papagelis Konstantinos, Galiotis Costas. Carbon nanotube-polymer composites: Chemistry, processing, mechanical and electrical properties, Progress in Polymer Science, 2010, Vol. 35, p. 357-401. (Eng.)

6. Hernandez-Montelongo J., Naveas N., Degoutin S., Tabary N., Chai F. , Spampinato V., Ceccone G., Rossi F., Torres-Costa V., Manso-Silvan M., Martel B. Porous siliconcyclodextrin based polymer composites for drug delivery applications, Carbohydrate Polymers, 2014, Vol. 110, p. 238-252. (Eng.)

7. Diaz-Bleis D., Vales-Pinzón C., Freile-Pelegrín Y., Alvarado-Gil J.J. Thermal characterization of magnetically aligned carbonyl iron/agar composites, Carbohydrate Polymers, 2014, Vol. 99, p. 84-90. (Eng.)

8. Samanvaya S., Jennifer L. Schaefer, Zichao Yang, Zhengyuan Tu, Lynden A. Archer. PolymerParticle Composites: Phase Stability and Applications in Electrochemical Energy Storage, Advansed Material, 2014, Vol. 26, p. 201-234. (Eng.)

9. Sathyanarayana S., Hübner $C$. Thermoplastic Nanocomposites with Carbon Nanotubes, Structural Nanocomposites, 2013, p. 19-60. (Eng.)

10. Huang Y. Y., Terentjev E. M. Dispersion of Carbon Nanotubes: Mixing, Sonication, Stabilization, and Composite Properties, Polymers, 2012, V. 4, p. 275-295. (Eng.)

11. Peng-Cheng Ma, Naveed A. Siddiqui, Gad Marom, Jang-Kyo Kim. Dispersion and functionalization of carbon nanotubes for polymer-based nanocomposites: A review, Composites: Part A, 2010, Vol. 41, p. 1345-1367. (Eng.)

12. Dinzhos R.V., Lysenkov E. A., Fialko N. M., Klepko $V$. $V$. Influence of the method of filling the filler on the thermophysical properties of systems based on thermoplastic polymers and carbon nanotubes, Physical engineering the surface, 2014, Vol.12, №4, p. 446-453. (Ukr.)

13. Malezhik A.V., Sementsov Yu.I., Yanchenko V.V. Synthesis of carbon nanotubes by catalytic decomposition, Journal of Applied Chemistry, 2005, Vol.78, p. 938-943. (Rus.)

14. Lemesh N.V., Lysenkov E.A., Gomza Yu.P. and etc. Structure of multilayered carbon nanotubes obtained by catalytic decomposition of ethylene on nickel nanoparticles, Ukrainian Chemical Journal, 2010, Vol. 76, №5, p. 29-36. (Rus.)

15. Giovanni A. L. A Steady-State Apparatus to Measure the Thermal Conductivity of Solids, Int. J. Thermophys, 2008, Vol. 29, p. 664-677. (Eng.) 